\title{
1 A chromosome-level genome assembly for the beet armyworm (Spodoptera exigua) using \\ 2 PacBio and Hi-C sequencing
}

3

$4 \quad$ Feng Zhang, Jianpeng Zhang, Yihua Yang and Yidong $\mathrm{Wu}^{*}$

5 Department of Entomology, College of Plant Protection, Nanjing Agricultural University, Nanjing 210095,

6 China

$7 \quad *$ Correspondence address. Yidong Wu, Nanjing University, Nanjing 210095, China. E-mail:

8 wyd@njau.edu.cn

9 


\section{Abstract}

\section{Background}

The beet armyworm, Spodoptera exigua (Hübner), is a worldwide, polyphagous agricultural pest feeding on vegetable, field, and flower crops. However, the lack of genome information on this insect severely limits our understanding of its rapid adaptation and hampers the development of efficient pest management strategies.

\section{Findings}

We report a chromosome-level genome assembly using single-molecule real-time PacBio sequencing and Hi-C data. The final genome assembly was $446.80 \mathrm{Mb}$ with a scaffold N50 of $14.36 \mathrm{Mb}$, and captured 97.9\% complete arthropod Benchmarking Universal Single-Copy Orthologs (BUSCO, $n=1,658$ ). A total of 367 contigs were anchored to 32 pseudo-chromosomes, covering $96.18 \%(429.74 \mathrm{Mb})$ of the total genome length. We predicted 17,727 protein-coding genes, of which $81.60 \%$ were supported by transcriptome evidence and $96.47 \%$ matched UniProt protein records. We also identified 867,102 (147.97 Mb/33.12\%) repetitive elements and 1,609 noncoding RNAs. Synteny inference indicated a conserved collinearity between three lepidopteran species. Gene family evolution and function enrichment analyses showed the significant expansions in families related to development, dietary, detoxification and chemosensory system, indicating these families may play a role in host plant specialization and niche adaptation.

\section{Conclusions}

We have generated a high-quality chromosomal-level genome that could provide a valuable resource for a better understanding and management of the beet armyworm.

Keywords: Noctuidae, agricultural pest, genome annotation, gene families, synteny

\section{Background}

Spodoptera exigua (NCBI:txid7107, Lepidoptera, Noctuidae), commonly known as the beet armyworm 
(BAW), is one of the world's most polyphagous pests, feeding on over 90 agricultural and horticultural plants. It heavily threatens agricultural economy and food security [1-3]. The beet armyworm has been distributed throughout the tropical and temperate regions of Asia, Europe, Africa and North America although it was native to Southeast Asia [4-6]. Severe outbreaks of this pest have been recorded in some region duo to complex factors, i.e. weather (rainfall, temperature), high-level resistance and increase of host planting $[\underline{5}, \underline{7}]$. Hence, S. exigua is considered an ideal model to study invasion events and population dynamics in agricultural pests $[3,4]$.

Currently, the use of chemical insecticides is the most widely adopted approach to suppress beet armyworm populations. However, surges in resistance to traditional insecticides (e.g. organophosphates, carbamates and pyrethroids), as well as newer insecticides with novel modes of action (e.g. emamectin benzoate, spinosad, abamectin), severely obstruct the continued use of insecticides [7, 8]. The advances in modern molecular techniques, in particular the CRISPR/Cas9 system [9, 10], have made it possible to explore the molecular functions of conserved insecticide target sites and their effect on resistance in $S$. exigua $[11,12]$. However, beyond these conserved target sites, it is difficult to uncover the molecular mechanisms of resistance starting from a phenotype alone, without some knowledge of the genome context and structure. Despite being an important pest, limited genomic resources have been available until now for the beet armyworm.

Here, we present a de novo chromosome-level genome assembly of S. exigua from a Chinese susceptible strain using single-molecule real-time (SMRT) Pacific Bioscience (PacBio) long reads and Hi-C data.

Mitochondrial genome and transcripts were also assembled. We annotated the essential genomic elements, i.e. repetitive sequences, protein-coding genes, and non-coding RNAs (ncRNAs). These valuable genomic data will help in the discovery of novel molecular mechanisms of resistance and will facilitate the design of future pest management strategies.

\section{Methods}

\section{Sample collection and sequencing}

The non-inbred strain WH-S, originally obtained from Wuhan Institute of Vegetables (Hubei, China) in 
2009 , has been maintained in the laboratory without exposure to any insecticides since its initial collection in 1998 from Wuhan [7]. Because the heterogeneity estimated from genome survey was not high (see below), WH-S strain was used for subsequent sequencing and genome assembly. Two female pupae, three adult males and six adult females were collected for sequencing experiments: one pupa for Illumina and PacBio whole genome, one pupa for Hi-C, and others for transcriptome, respectively. Genomic DNA/RNA extraction, library preparation and sequencing were carried out at BGI (Shenzhen, China) except for Hi-C library. Genomic DNA was extracted using the Qiagene Blood \& CELL Culture DNA mini Kit. Long read libraries were constructed with an insert size of $15 \mathrm{~kb}$ using a SMRTbell DNA Template Prep Kit 1.0 and sequenced on a PacBio Sequel system (RRID:SCR_017989) using Sequel Sequencing kit v2.1 chemistry. Short read libraries of an insert size of 350 bp were prepared using the TruSeq DNA PCR-Free LT Library Preparation Kit and were subject to the paired-end 150 bp (PE 150) sequencing on the HiSeq X Ten platform (RRID:SCR_016385). Hi-C library preparation, including cross-linking the DNA, restriction enzyme digestion, end repair, DNA cyclization, and DNA purification, was performed by Frasergen Co. Ltd. (Wuhan, China). Restriction Enzyme MboI was used to digest DNA for the Hi-C assay. Genomic RNA was extracted using TRIzol ${ }^{\mathrm{TM}}$ Reagent and converted into libraries using the TruSeq RNA v2 Kit.

\section{Genome assembly}

Quality control was performed for the raw Illumina short reads using BBTools suite v38.49 [13]: duplicates were removed using clumpy.sh; low-quality bases were trimmed using bbduk.sh, i.e. a minimum Phred quality score of 20 for both read sides, a minimum read/Ns length of $15 / 5 \mathrm{bp}$, a maximum length polyA/G/C tail of $10 \mathrm{bp}$. Preliminary genome survey was investigated employing the strategy of short-read kmer distributions using GenomeScope v1.0.0 (GenomeScope, RRID:SCR_017014) [14]. The histogram of k-mer frequencies was estimated with 21-mers using khist.sh (BBTools). Estimated genome sizes increased with the parameter of maximum k-mer coverage cutoff, thus we selected 1,000 and 10,000 as the maximum k-mer coverage cutoffs.

The raw PacBio long reads were assembled into contigs using Flye v2.5 (Flye, RRID:SCR_017016) [15] and Falcon v1.3.0 (Falcon, RRID:SCR_016089) [16]; both assemblies were self-polished with a round of Flye polishing module "--polish-target”. To improve contig contiguity, Flye and Falcon assemblies were 
merged with two rounds of quickmerge v.0.3 [17] following USAGE 2 [18]; Falcon assembly was treated as the query in both rounds because of its higher contiguity (greater N50 value); Flye assembly was treated as the reference in the first round and the merged assembly from the first round was set as the reference in the second round; for each round, the N50 length of the reference assembly was assigned to the length cutoff for anchor contigs ('-1'). We removed redundant heterozygous contigs with Purge Haplotigs v1.1.0 [19]; percent cutoff for identifying a contig as a haplotig was set as 50 (-a 50) with other parameters as the default. Non-redundant assembly was further polished with Illumina short reads using two rounds of Pilon v1.22 (Pilon, RRID:SCR_014731 [20]; input BAM mapping files were generated using Minimap2 v2.17 (Minimap2, RRID:SCR_018550) [21] and SAMtools v1.9 (Samtools, RRID:SCR_002105) [22].

To perform the chromosome-level concatenation, we aligned Hi-C read pairs to the genome, removed duplicates, and extracted the Hi-C contacts using Juicer v1.6.2 (Juicer, RRID:SCR_017226) [23]. Pseudochromosomes were generated using 3D-DNA v180922 [24] in combination with a review step in Juicebox Assembly Tools module within Juicebox [23]. Sex chromosomes with female heterogamety were determined according to male-to-female comparison approach [25]. In theory, the average depth of sex chromosomes should be about half of that for autosomes in females, and the average depth of $Z$ chromosome should be approximately equal to autosomes whereas $\mathrm{W}$ chromosome should nearly be zero in males. Firstly, we mapped Illumina short reads to the genome assembly using Minimap2 [21], then the average depth of coverage was calculated using SAMtools [22]. In fact, the average depth of coverage of W chromosome is higher than expected because harboring genes may be similar to some regions of autosomes and $\mathrm{Z}$ chromosomes [25]. Chromosomal nomenclature order and orientation were assigned in comparison with Bombyx mori genome [26].

Contaminants were removed against the nt and UniVec databases using HS-BLASTN v0.0.5 [27] and BLAST+ (i.e. blastn) v2.7.1 (BLASTN, RRID:SCR_001598) [28]. We assessed the assembly completeness using Benchmarking Universal Single-Copy Orthologs (BUSCO, RRID:SCR_015008) [29] analyses against insect reference dataset (insect_odb9, $\mathrm{n}=1,658$ ). We also mapped PacBio long reads and Illumina short reads to the final genome assembly with Minimap2. 


\section{Genome annotation}

Repetitive elements, ncRNAs and protein-coding genes were annotated for the beat armyworm. To identify repeat elements, we constructed a de novo species specific repeat library using RepeatModeler v1.0.11 (RepeatModeler, RRID:SCR_015027) [30], and then combined it with Dfam_3.0 [31] and RepBase20181026 databases [32] to generate a custom library. We then used RepeatMasker v4.0.9 (RepeatMasker, RRID:SCR_012954) [33] to mask repeats in the genome assembly with the combined custom library. Noncoding RNAs were identified with Infernal v1.1.2 (Infernal, RRID:SCR_011809) [34] and tRNAscan-SE v2.0 (tRNAscan-SE, RRID:SCR_010835) [35].

Protein-coding genes were predicted with the MAKER v2.31.10 pipeline (MAKER, RRID:SCR_005309) [36] which can integrate ab initio, transcriptome- and protein homology-based evidence. For the ab initio gene predictions, we trained gene model parameters for Augustus v3.3 (Augustus, RRID:SCR_008417) [37] and GeneMark-ET v4.38 (GeneMark, RRID:SCR_011930) [38] using BRAKER v2.1.0 [39], which incorporated RNA-seq data to accurately identify exon/intron boundaries. Input BAM alignment file for BRAKER was generated with HISAT2 v2.1.0 (HISAT2, RRID:SCR_015530) [40]. For the transcriptome-based evidence, a genome-guided method was applied to transcript assembly using StringTie v1.3.4 (StringTie, RRID:SCR_016323) [41]. For the protein homologybased evidence, protein sequences of Apis mellifera (GCF_003254395.2), Bombyx mori (GCF_000151625.1), Drosophila melanogaster (GCF_000001215.4), Tribolium castaneum (GCF_000002335.3), Helicoverpa armigera (GCF_002156985.1) and Spodoptera litura (GCF_002706865.1) were downloaded from the National Center for Biotechnology Information (NCBI) [42]. Finally, repeat-masked genome and repeat annotations, assembled transcripts, protein sequences, and ab initio predicted gene models from BRAKER were passed to MAKER for the gene structure predictions. Gene functions were assigned to MAKER predicted gene models using Diamond v0.9.24 (DIAMOND, RRID:SCR_016071) [43] against the UniProtKB (UniProtKB, RRID:SCR_004426) database [44] with the sensitive mode '--more-sensitive -e 1e-5'. We searched protein domains, Gene Ontology (GO) and Reactome pathway using InterProScan 5.41-78.0 (InterProScan, RRID:SCR_005829) [45] against Pfam (Pfam, RRID:SCR_004726) [46], Gene3D (Gene3D, RRID:SCR_007672) [47], Superfamily 

v2.0 [51] against the eggNOG v5.0 (eggNOG, RRID:SCR_002456) database [52] to obtain additional information of GO, Kyoto Encyclopedia of Genes and Genomes (KEGG, RRID:SCR_012773) orthology (ko), KEGG pathways, enzyme codes (ECs), and clusters of orthologous groups (COG,

RRID:SCR_007139). Circus figure showing the element tracks was produced by Tbtools v1.0 [53].

\section{Gene family evolution}

We selected 14 species for orthogroup inference, including Diptera (Drosophila melanogaster), Trichoptera (Stenopsyche tienmushanensis), Plutellidae (Plutella xylostella), Pyralidae (Amyelois transitella), Nymphalidae (Danaus plexippus), Geometridae (Operophtera brumata), Sphingidae (Manduca sexta), Bombycidae (Bombyx mori), Noctuidae (Helicoverpa armigera, Heliothis virescens, Spodoptera exigua, Spodoptera frugiperda, Spodoptera litura, Trichoplusia ni). Lepidoptera taxa covered a butterfly and six representative pest families. All protein sequences were downloaded from the NCBI except for Stenopsyche tienmushanensis from GigaScience Database [54]. Orthogroups were inferred using OrthoFinder v2.3.8 (OrthoFinder, RRID:SCR_017118) [55] with the Diamond as the sequence aligner.

Phylogenomic reconstructions were performed using single-copy orthologous inferred from OrthoFinder. Protein sequences of each orthogroup were aligned using MAFFT v7.394 (MAFFT, RRID:SCR_011811) [56] with the L-INS-I strategy, trimmed using trimAl v1.4.1 (trimAl, RRID:SCR_017334) [57] with the heuristic method 'automated1', and concatenated using FASconCAT-G v1.04 [58]. Phylogenetic tree was reconstructed using IQ-TREE v2.0-rc1 (IQ-TREE, RRID:SCR_017254) [59] with the substitution model restricted to the LG model and $10 \%$ of partition pairs for partition clustering algorithm to reduce the computational burden ('-m MFP --mset LG --msub nuclear --rclusterf 10’). Genes that violate models, i.e. genes undergoing non-SRH (stationary, reversible, and homogeneous) evolution, were also removed prior to tree inference ('--symtest-remove-bad --symtest-pval 0.10 '). Node support values were calculated ('-B 1000 --alrt 1000') using 1,000 SH-aLRT replicates [60] and 1,000 ultrafast bootstraps [61]. Divergence time was inferred using MCMCTree within the PAML v4.9j (PAML, RRID:SCR_014932) package [62]. 
Four fossil calibrations were extracted from the Paleobiology Database [63] for dating: root (314.6-318.1 Mya), Trichoptera (311.4-314.6 Mya), Ditrysia (93.5-145.2 Mya) and Noctuoidea (28.1-33.9 Mya).

Expansions and contractions of gene families were estimated using CAFÉ v4.2.1 [64] using the method of single birth-death parameter lambda with the significance level of 0.01 . Function enrichment analyses of GO and KEGG categories were also performed for those significantly expanded families using R package clusterProfiler v3.10.1 (clusterProfiler, RRID:SCR_016884) [65] with the default significance values (pvalue as 0.01 and q-value as 0.05$)$.

\section{Genome synteny}

To search for syntenic blocks between genomes, coding sequences (CDSs) of S. exigua (except for the W chromosome) and other two species (B. mori, S. litura) were used for analyses. Chromosome W was not included because S. litura genome did not assign any contigs into this chromosome. Chromosome-level genomic resources were downloaded from the NCBI (S. litura, GCF_002706865.1) and SilkBase v2.1 (B. mori) [66]. We searched pairwise synteny using LAST v1080 (LAST, RRID:SCR_006119) [67] and removed tandem duplications and weak hits by the module McScan (MCScan, RRID:SCR_017650) [68] (Python version) in jcvi [69]. We extracted subset of blocks with following options “--minspan=30 -minsize=15 --simple". Finally, the high-quality syntenic blocks were visualized by Circos v0.69-9 (Circos, RRID:SCR_011798) [70].

\section{Results}

\section{Genome assembly}

We generated 3,919,515 PacBio subreads of $38.98 \mathrm{~Gb}(87 \mathrm{X})$ with the mean and N50 length reaching 9.95 and $16.01 \mathrm{~kb}$, respectively. A total of $34.62 \mathrm{~Gb}(77 \mathrm{X}), 65.21 \mathrm{~Gb}$ and $56.32 \mathrm{~Gb}(126 \mathrm{X})$ were produced in the Illumina X Ten platform for whole genome, transcriptome and Hi-C sequencing, respectively. Estimated genome size ranged from $408.58 \mathrm{Mb}$ to $448.90 \mathrm{Mb}$ (Table $\mathrm{S} 1$ ). Genome repeat length increased with the maximum k-mer coverage cutoff, ranging from 64.96 Mb to $104.93 \mathrm{Mb}$. Heterozygosity rate $(0.585-0.594)$ and a small indistinct peak on the left of main peak (Fig. 1a) implied that the heterozygosity of the 
sequenced strain was not high.

Both Flye and Falcon assemblies (Table S2) were ca. $100 \mathrm{Mb}$ larger than the estimated size due to a lowmoderate rate of heterozygosity (duplicated BUSCOs $>10 \%$ ), and merged into a $561.68 \mathrm{Mb}$-assembly of 934 contigs (N50 length $3.47 \mathrm{Mb}$ ). We removed 668 redundant heterozygous sequences using Purge Haplotigs, resulting an obvious improvement of N50 length (5.53 Mb). After polishing, chromosome concatenation and contaminant removal, the final genome assembly had 301 scaffolds/667 contigs, a total length of $446.80 \mathrm{Mb}$, scaffold/contig N50 length of $14.36 / 3.47 \mathrm{Mb}$, and a GC content of $36.67 \% ; 367$ contigs were concatenated into 32 pseudo-chromosomes (Fig. 1b), accounting for $96.18 \%$ (429.74 Mb) of the total genome length. Through 3D-DNA pipeline, partial assembled scaffolds or contigs in previous assembly process were split into shorter sequences due to the misjoints. We successfully determined 30 autosomes, $\mathrm{Z}$ and $\mathrm{W}$ chromosome with their nomenclature order and orientation homologous to those of $B$. mori (Fig. 1c). The final assembled size was very close to the estimated size from GenomeScope (Table S1). BUSCO completeness analyses against insect_odb9 reference dataset $(n=1,658)$ (Table S2) showed our assembled versions covered $97.8-98.6 \%$ complete, $0.2-0.4 \%$ fragmented, and $1.1-1.9 \%$ missing BUSCO genes. We mapped $97.62 \%$ and $93.78 \%$ Illumina short and PacBio long reads to our final assembly. All these assessments showed the accuracy and the high integrity of our final genome assembly with low redundancy ( $2.1 \%$ duplicated BUSCOs). Among four Noctuidae pest species (Table 1), assemblies of S. exigua and S. frugiperda have the highest genome contiguity (contig N50>1 Mb, contig number $<1,000)$ and the lowest gap content $(<0.1 \%)$.

TABLE 1: Genome assembly statistics of four Noctuidae species available on GenBank

\begin{tabular}{lllll}
\hline Characteristics & $\begin{array}{l}\text { Spodoptera } \\
\text { exigua }\end{array}$ & $\begin{array}{l}\text { Spodoptera } \\
\text { litura }\end{array}$ & $\begin{array}{l}\text { Spodoptera } \\
\text { frugiperda }\end{array}$ & $\begin{array}{l}\text { Helicoverpa } \\
\text { armigera }\end{array}$ \\
\hline Accession number & GCA_011316535.1 & GCA_002706865.1 & GCA_011064685.1 & GCA_002156985.1 \\
Assembly size (Mb) & 446.80 & 438.96 & 486.29 & 337.09 \\
Number of scaffolds & 301 & 2,975 & 92 & 998 \\
Longest scaffold (Mb) & 19.74 & 18.94 & 22.37 & 6.15 \\
Scaffold N50 length (Mb) & 14.36 & 13.59 & 16.35 & 1.00 \\
\hline
\end{tabular}




\begin{tabular}{lllll}
\hline Number of contigs & 667 & 13,637 & 617 & 24,552 \\
Longest contig (Mb) & 15.88 & 0.64 & 16.14 & 0.31 \\
Contig N50 length (Mb) & 3.47 & 0.068 & 1.13 & 0.023 \\
Pseudo-chromosomes & 32 & 31 & 32 & 0 \\
GC (\%) & 36.67 & 36.56 & 36.43 & 37.50 \\
Gaps (\%) & 0.075 & 2.49 & 0.011 & 11.01 \\
BUSCO completeness (\%) & 97.9 & 97.1 & 93.4 & 96.8 \\
\hline
\end{tabular}

\section{Genome annotation}

By combing de novo and homology-based evidence, we identified that $33.12 \%$ (147.97 $\mathrm{Mb})$ of the genome was composed of repetitive elements in the beet armyworm genome. Repeat ratio was very close to the repeat content $(31.8 \%)$ in the published S. litura genome (Cheng et al. 2017). The main interspersed repeat families were LINE (14.81\%), Unknown (5.89\%), RC (3.95\%), LTR (3.10\%), SINE (2.53\%), and DNA (1.79\%). Simple repeats also occupied $0.83 \%$ of the genome (Table S3). Interestingly, chromosome W had more abundant DNA transposons and LTR retrotransposons but fewer interspersed nuclear elements (SINEs and LINEs) compared to other chromosomes (Fig. 1c).

We identified 172 rRNAs (5S, 5.8S, LSU, SSU), 61 miRNAs (37 families), 118 small nuclear RNAs (snRNAs, 19 families), 2 long non-coding RNAs (lncRNA-Sphinx), 1,135 transfer RNAs (tRNAs), 3 ribozymes (2 families), 114 cis-regulatory elements, and 3 other (Metazoa_SRP) ncRNAs (Table S4). Twenty-one tRNA isotypes were identified except for SelCys-isotype which was lacking. Small nuclear RNAs were classified into six spliceosomal RNAs (U1, U2, U4, U5, U6, U11), three minor spliceosomal RNAs (U12, U4atac, U6atac), and four H/ACA box and 20 C/D box small nucleolar RNAs (snoRNAs). We predicted 17,727 protein-coding genes (gene models) with a mean length of 9,604.99 bp in the beet armyworm genome. BUSCO completeness assessment using the protein mode ('-m prot') identified 1,601 (96.6\%) complete, 45 (2.7\%) duplicated, 12 (0.7\%) fragmented, and 45 (2.7\%) missing BUSCO genes, indicating the highly completeness of our predicted protein-coding gene set. Exons and introns had a mean count of 6.40 and 5.20 per gene and a mean length of $334.78 \mathrm{bp}$ and 1,434.55 bp, respectively (Table 2). Among predicted genes, $81.60 \%$ were supported by transcriptome evidence and $96.47 \%$ matched the 

genes, respectively.

TABLE 2: Genome annotation statistics of the beet armyworm

\begin{tabular}{ll}
\hline Elements & Number \\
\hline Protein-coding genes & 17,727 \\
Mean protein (aa) and gene (bp) length & $482.19 / 9,604.99$ \\
Exons/introns per gene & $6.40 / 5.20$ \\
Exon & $37.95 \mathrm{Mb}(8.49 \%)$ \\
Intron & $132.31 \mathrm{Mb}(28.34 \%)$ \\
Mean exon/intron length & $334.78 / 1,434.55$ \\
BUSCO completeness (\%) & 96.6 \\
GO & 10,298 \\
Reactome pathway & 2,731 \\
KEGG ko & 7,643 \\
& \\
KEGG pathway & 2,675 \\
& \\
\hline
\end{tabular}




\section{Gene family evolution}

We assigned a total of $95.59 \%(214,213)$ genes into 15,906 orthogroups (gene families) using OrthoFinder. Among them, 4,618 orthogroups were shared by all fourteen species and 1,215 are single-copy ones; 62 families and 586 orthologs are common to six Noctuidae species; 2,376 orthogroups and 9,324 orthologs were species-specific. For the beat armyworm, 17,094 (96.43\%) genes were assigned into 11,663 orthogroups; 154 families and 569 genes were species-specific (Fig. 2a, Table S5).

For phylogenomic analyses, we removed 109 single-copy loci that violated SRH hypothesis, resulting a matrix of 1,106 loci and 456,865 amino acid sites. Our phylogeny was largely consistent with the newest lepidopteran classification [71] except for the position of Operophtera brumata (Geometridae), which was clustered to Noctuoidea than Bombycoidea. Very weak node support (14.4/41) also confirmed the unstable position of this species (Fig. 2a). Divergence time estimation showed that Noctuidae diverged 32.58 34.47 Mya, conforming to the oldest Noctuidae fossil (Noctuites incertissima, 28.1 33.9 Mya) [72]. The bear armyworm diverged from the other two Spodoptera species 7.40 8.47 Mya.

We identified 974/2,059 expanded/contracted gene families in S. exigua genome (Fig. 2a), 278 and 120 of them being rapidly evolving at the significant level of 0.01 . Haemolymph juvenile hormone binding protein (JHBP), chorion protein and insect cuticle protein have a profound effect in regulating embryogenesis, maintaining the status quo of larva development and stimulating adult reproductive maturation [73-75]. Gustatory receptors (GRs, referred as 7tm chemosensory receptor in Fig. 2b) were also found to be expanded in sampled 14 species (Fig. 2b). Massive expansion of GRs in Lepidoptera may be related to extreme polyphagy $[76,77]$. S. exigua feeds a large number of host plants including more than 40 monocot families [78]. Trypsin and cytochrome P450 (CYP, not shown in Fig. 2b) plays an important role in digestion [79] and detoxification [80] by contributing to protein digestion, xenobiotic metabolism, insecticide resistance, odorant or pheromone metabolism. In addition, many gene families are transposon repeat-related, such as reverse transcriptase, DDE superfamily endonuclease, Baculovirus FP protein, transposase, integrase core domain-containing protein, retrotransposon gag protein, phage integrase etc. Large expansions of these transposable elements explain the relatively high ratio of repetitive elements, particularly LINE superfamilies CR1-Zenon, L2, R1 and RTE (Table S3), although their functions are 
largely unknown.

$$
\text { GO and KEGG function of rapidly expanded families furthermore refined above hypotheses. The top six }
$$
GO enrichment categories, i.e. GO term count greater than 25, related to transposon and egg chorion (Fig. 3a). KEGG pathways (Fig. 3b) were enriched in development (folate biosynthesis), digestion (cholesterol metabolism, lysosome, arachidonic acid metabolism, carbohydrate digestion and absorption), and detoxification (xenobiotic and drug metabolism by CYP, glutathione metabolism). Gene family evolution provides valuable information supporting genetic mechanisms of polyphagy and ecological adaptions for the beet armyworm.

\section{Synteny}

Syntenic analyses showed that 100 syntenic blocks between S. exigua and B. mori and 148 syntenic blocks between S. exigua and S. litura were conserved. Three B. mori chromosomes 11, 23 and 24 were clearly divided into three pairs in S. exigua: 11 and 29, 23 and 30, 24 and 31, respectively (Fig. 4a). Strong syntenic correspondence between S. exigua and S. litura (Fig. 4b) indicated the conserved chromosomelevel gene collinearity, which was also reported previously [81, 82].

\section{Availability of Supporting Data and Materials}

The project and raw sequencing data have been deposited at the NCBI under the accessions PRJNA588360 and SRR10441878-SRR10441889. The data sets supporting the results of this article are available in the GigaScience GigaDB repository.

\section{Additional Files}

Supplementary Table S1: Genome survey for Spodoptera exigua.

Supplementary Table S2: Summary of each assembly version for Spodoptera exigua.

Supplementary Table S3: Repeat annotation in Spodoptera exigua.

Supplementary Table S4: Summary statistics for non-coding RNAs in Spodoptera exigua. 
Supplementary Table S5: Summary statistics for gene family inference.

\section{Abbreviations}

BAM: binary alignment map; BLAST: Basic Local Alignment Search Tool; bp: base pairs; BUSCO: benchmarking universal single-copy orthologs; CDS: coding sequence; COG: clusters of orthologous group; CYP: cytochrome P450; EC: enzyme codes; Gb: gigabase pairs; GC: guanine-cytosine; GO: gene ontology; GR: gustatory receptor; Hi-C: chromosome conformation capture; kb: kilobase pairs; KEGG: Kyoto Encyclopedia of Genes and Genomes; ko: KEGG orthology; LINE: long interspersed nuclear element; lncRNA: long non-coding RNA; LSU: large subunit rRNA; LTR: long terminal repeat; Mb: megabase pairs; miRNA: micro RNA; ncRNA: non-coding RNA; NCBI: National Center for Biotechnology Information; PacBio: Pacific BioSciences; PBDB: Paleobiology Database; PE: paired end; RC: rolling circle; RNA-seq: RNA sequencing; rRNA: ribosomal RNA; SINE: short interspersed nuclear element; SMRT: single-molecule real time; snRNA: small nuclear RNA; snoRNA: small nucleolar RNA; SRA: Sequence Read Archive; SSU: small subunit rRNA; tRNA: transfer RNA.

\section{Competing Interests}

All authors declare that they have no competing interests.

\section{Funding}

This work was supported by a grant (no. 31572030) from the National Natural Science Foundation of China and the Fundamental Research Funds for the Central Universities (KYZ201920).

\section{Authors' Contributions}

Y.W. and Y.Y. conceived this study. F.Z. and J.Z. carried out the experiments and performed the analyses. F.Z. and Y.W. took the lead in writing the manuscript. All authors discussed the results and contributed to the final manuscript. 
bioRxiv preprint doi: https://doi.org/10.1101/2019.12.26.889121; this version posted August 14, 2020. The copyright holder for this preprint (which was not certified by peer review) is the author/funder, who has granted bioRxiv a license to display the preprint in perpetuity. It is made available under aCC-BY-NC 4.0 International license. 


\section{References}

1. Mehrkhou F, Talebi AA, Moharramipour S, et al. Demographic parameters of Spodoptera exigua (Lepidoptera: Noctuidae) on different soybean cultivars. Environ Entomol 2012;41:326-32.

2. Saeed Q, Ahmad F, Iqbal N, et al. Chemical control of polyphagous pests on their auxiliary hosts can minimize insecticide resistance: A case study of Spodoptera exigua Hübner (Lepidoptera: Noctuidae) in cotton agroecosystem. Ecotox Environ Safety 2019;171:721-7.

3. Tay WT, Gordon KHJ. Going global - genomic insights into insect invasions. Curr Opin Insect Sci 2019;31:123-30.

4. Han LZ, Gu HN, Zhai BP, et al. Genetic effects on flight capacity in the beet armyworm, Spodoptera exigua (Lep., Noctuidae). J Appl Entomol 2009;133:262-71.

5. Zhou LH, Wang XY, Xu GQ, et al. Mitochondrial DNA phylogeography of Spodoptera exigua across a broad geographic area in China. J Appl Entomol 2017;141:527-39.

6. Fu X, Feng H, Liu Z, et al. Trans-regional migration of the beet armyworm, Spodoptera exigua (Lepidoptera: Noctuidae), in North-East Asia. PLoS One 2017;12:e0183582.

7. Che W, Shi T, Wu Y, et al. Insecticide resistance status of field populations of Spodoptera exigua (Lepidoptera: Noctuidae) from China. J Econ Entomol 2013;106:1855-62.

8. Ishtiaq M, Saleem MA. Generating susceptible strain and resistance status of field populations of Spodoptera exigua (Lepidoptera: Noctuidae) against some conventional and new chemistry insecticides in Pakistan. J Econ Entomol 2011;104:1343-8.

9. Cong L, Ran FA, Cox D, et al. Multiplex genome engineering using CRISPR/Cas systems. Science 2013;339:819-823.

10. Bassett AR, Tibbit C, Ponting CP, et al. Highly efficient targeted mutagenesis of Drosophila with the CRISPR/Cas9 system. Cell Rep 2013;4:220-8.

11. Zuo Y, Wang H, Xu Y, et al. CRISPR/Cas9 mediated G4946E substitution in the ryanodine receptor of Spodoptera exigua confers high levels of resistance to diamide insecticides. Insect Biochem Mol Biol 2017;89:79-85.

12. Zuo YY, Huang JL, Wang J, et al. Knockout of a P-glycoprotein gene increases susceptibility to 
abamectin and emamectin benzoate in Spodoptera exigua. Insect Mol Biol 2018;27:36-45.

13. Bushnell B. BBtools. 2014. https://sourceforge.net/projects/bbmap/.

14. Vurture GW, Sedlazeck FJ, Nattestad M, et al. GenomeScope: fast reference-free genome profling from short reads. Bioinformatics 2017;33:2202-4.

15. Kolmogorov M, Yuan J, Lin Y, et al. Assembly of long, error-prone reads using repeat graphs. Nat Biotechnol 2019;37:540-6.

16. Chin CS, Peluso P, Sedlazeck FJ, et al. Phased diploid genome assembly with single-molecule realtime sequencing. Nat Methods 2016;13:1050-4.

17. Chakraborty M, Baldwin-Brown JG, Long AD, et al. Contiguous and accurate de novo assembly of metazoan genomes with modest long read coverage. Nucleic Acids Res 2016;44:e147.

18. Chakraborty M. How to use Quickmerge: A guide. https://github.com/mahulchak/quickmerge/wiki. Accessed 1 September 2019.

19. Roach MJ, Schmidt SA, Borneman AR. Purge Haplotigs: allelic contig reassignment for third-gen diploid genome assemblies. BMC Bioinformatics 2018;19:460.

20. Walker BJ, Abeel T, Shea T, et al. Pilon: an integrated tool for comprehensive microbial variant detection and genome assembly improvement. PLoS ONE 2014;9:e112963.

21. Li H. Minimap2: pairwise alignment for nucleotide sequences. Bioinformatics 2018;34:3094-100.

22. Li H, Handsaker B, Wysoker A, et al. The Sequence alignment/map SAM format and SAMtools, Bioinformatics 2009;2516:2078-9.

23. Durand NC, Shamim MS, Machol I, et al. Juicer provides a one-click system for analyzing loop-

26. Kawamoto M, Jouraku A, Toyoda A, et al. High-quality genome assembly of the silkworm, Bombyx resolution Hi-C Experiments. Cell Syst 2016;3:95-8.

24. Dudchenko O, Batra SS, Omer AD, et al. De novo assembly of the Aedes aegypti genome using Hi-C yields chromosome-length scaffolds. Science 2017;356:92-5.

25. Tomaszkiewicz M, Medvedev P, Makova KD. Y and W chromosome assemblies: approaches and discoveries. Trends Genet 2017;33:266-82. mori. Insect Biochem Mol Biol 2019;107:53-62.

27. Chen Y, Ye W, Zhang Y, et al. High speed BLASTN: an accelerated MegaBLAST search tool. Nucleic 
Acids Res 2015;43:7762-8.

28. Camacho C, Coulouris G, Avagyan V, et al. BLAST+: architecture and applications. BMC Bioinformatics 2009;10:421.

29. Waterhouse RM, Seppey M, Simão FA, et al. BUSCO Applications from Quality Assessments to Gene Prediction and Phylogenomics. Mol Biol Evol 2018;35:543-8.

30. Smit AFA, Hubley R. RepeatModeler Open-1.0. 2008-2015. http://www.repeatmasker.org.

31. Hubley R, Finn RD, Clements J, et al. The Dfam database of repetitive DNA families. Nucleic Acids Res 2016;44:D81-9.

32. Bao W, Kojima KK, Kohany O. Repbase Update, a database of repetitive elements in eukaryotic genomes. Mobile DNA 2015;6:11.

33. Smit AFA, Hubley R, Green P. RepeatMasker Open-4.0. 2013-2015. http://www.repeatmasker.org.

34. Nawrocki EP, Eddy SR. Infernal 1.1: 100-fold faster RNA homology searches. Bioinformatics 2013;29:2933-5.

35. Chan PP, Lowe TM. tRNAscan-SE: Searching for tRNA Genes in Genomic Sequences. Method Mol Biol 2019;1962:1-14.

36. Holt C, Yandell M. MAKER2: an annotation pipeline and genome-database management tool for second-generation genome projects. BMC Bioinformatics 2011;12:491.

37. Stanke M, Steinkamp R, Waack S, et al. AUGUSTUS: a web server for gene finding in eukaryotes. Nucleic Acids Res 2004;32;W309-12.

38. Lomsadze A, Ter-Hovhannisyan V, Chernoff YO, et al. Gene identification in novel eukaryotic genomes by self-training algorithm. Nucleic Acids Res 2005;33;6494-506.

39. Hoff KJ, Lange S, Lomsadze A, et al. BRAKER1: Unsupervised RNA-Seq-Based Genome Annotation with GeneMark-ET and AUGUSTUS. Bioinformatics 2016;32:767-9.

40. Kim D, Langmead B, Salzberg SL. HISAT: a fast spliced aligner with low memory requirements. Nat Methods 2015;12:357-60.

41. Pertea M, Pertea GM, Antonescu CM, et al. StringTie enables improved reconstruction of a transcriptome from RNA-seq reads. Nat Biotechnol 2015;33:290-5.

42. National Center for Biotechnology Information NCBI. https: //www.ncbi.nlm.nih.gov. Accessed April 
2020.

43. Buchfink B, Xie C, Huson DH. Fast and sensitive protein alignment using DIAMOND. Nat Methods 2015;12:59-60.

44. The UniProt Consortium. UniProt: a worldwide hub of protein knowledge. Nucleic Acids Res 2019;47:D506-15.

45. Finn RD, Attwood TK, Babbitt PC, et al. InterPro in 2017-beyond protein family and domain annotations. Nucleic Acids Res 2017;45:D190-9.

46. El-Gebali S, Mistry J, Bateman A, et al. The Pfam protein families database in 2019. Nucleic Acids Res 2019;47:D427-2.

47. Lewis TE, Sillitoe I, Dawson N, et al. Gene3D: extensive prediction of globular domains in proteins. Nucleic Acids Res 2018;46:D435-9.

48. Wilson D, Pethica R, Zhou Y, et al. SUPERFAMILY — sophisticated comparative genomics, data mining, visualization and phylogeny. Nucleic Acids Res 2009;37:D380-6.

49. Marchler-Bauer A, Bo Y, Han L, et al. CDD/SPARCLE: functional classification of proteins via subfamily domain architectures. Nucleic Acids Res 2017;45:D200-3.

50. Letunic I, Bork P. 20 years of the SMART protein domain annotation resource. Nucleic Acids Res 2017;46:D493-6.

51. Huerta-Cepas J, Forslund K, Coelho LP, et al. Fast genome-wide functional annotation through orthology assignment by eggNOG-Mapper. Mol Biol Evol 2017;34:2115-22.

52. Huerta-Cepas J, Szklarczyk D, Heller D, et al. eggNOG 5.0: a hierarchical, functionally and phylogenetically annotated orthology resource based on 5090 organisms and 2502 viruses. Nucleic Acids Res 2019;47:D309-14.

53. Chen C, Chen H, Zhang Y, et al. TBtools: an integrative toolkit developed for interactive analyses of big biological data. Mol Plant 2020;138:1194-202.

54. Luo S, Tang M, Frandsen PB, et al. Supporting data for "The genome of an underwater architect, the caddisfly Stenopsyche tienmushanensis Hwang (Insecta: Trichoptera).” GigaScience Database. 2018. http://dx.doi.org/10.5524/100538.

55. Emms DM, Kelly S. OrthoFinder: phylogenetic orthology inference for comparative genomics. 
Genome Biol 2019;20:238.

56. Katoh K, Standley DM. MAFFT multiple sequence alignment software version 7: Improvements in performance and usability. Mol Biol Evol 2013;30:772-80.

57. Capella-Gutiérrez S, Silla-Martínez JM, Gabaldón T. trimAl: a tool for automated alignment trimming in large-scale phylogenetic analyses. Bioinformatics 2009;25:1972-3.

58. Kück P, Longo GC. FASconCAT-G: extensive functions for multiple sequence alignment preparations concerning phylogenetic studies. Front Zool 2014;11:81.

59. Minh BQ, Schmidt HA, Chernomor O et al. IQ-TREE 2: New models and efficient methods for phylogenetic inference in the genomic era. Mol Biol Evol 2020;37:1530-4.

60. Guindon S, Dufayard JF, Lefort V, et al. New algorithms and methods to estimate maximum-likelihood phylogenies: Assessing the performance of PhyML 3.0. Syst Biol 2010;59:307-21.

61. Hoang DT, Chernomor O, von Haeseler A, et al. UFBoot2: Improving the ultrafast bootstrap approximation. Mol Biol Evol 2018;35:518-22.

62. Yang Z. PAML 4: phylogenetic analysis by maximum likelihood. Mol Biol Evol 2007;24:1586-91.

63. PBDB Navigator. https://www.paleobiodb.org/navigator/. Accessed 20 May 2020.

64. Han MV, Thomas GW, Lugo-Martinez J, et al. Estimating gene gain and loss rates in the presence of error in genome assembly and annotation using CAFE 3. Mol Biol Evol 2013;308:1987-97.

65. Yu G, Wang L, Han Y, et al. clusterProfiler: an R package for comparing biological themes among gene clusters. OMICS 2012;165:284-7.

66. SilkBase. http://silkbase.ab.a.u-tokyo.ac.jp. Accessed 20 May 2020.

67. Kiełbasa SM, Wan R, Sato K, et al. Adaptive seeds tame genomic sequence comparison. Genome Res 2011;21:487-93.

68. Tang H, Bowers JE, Wang X, et al. Synteny and collinearity in plant genomes. Science 2008;320:4868.

69. Tang H, Krishnakumar V, Li J, et al. jcvi: JCVI utility libraries. Zenodo 2015; doi:10.5281/zenodo.31631.

70. Krzywinski M, Schein J, Birol I, et al. Circos: an information aesthetic for comparative genomics. Genome Res 2009;19:1639-45. 
71. Kawahara AY, Plotkin D, Espeland M, et al. Phylogenomics reveals the evolutionary timing and pattern of butterflies and moths. Proc Natl Acad Sci USA. 2019;11645:22657-63.

72. Oustalet E. Recherches sur les insectes fossiles des terrains Tertiaires de la France, première partie, insectes fossiles de l'Auvergne. Ann Sci Géol, Paris 1870;23:1-178

73. Waring GL. Morphogenesis of the eggshells in Drosophila. Int Rev Cytol 2000;198:67-108.

74. Kolodziejczyk R, Kochman M, Bujacz G, et al. Crystallization and preliminary crystallographic studies of juvenile hormone-binding protein from Galleria mellonella haemolymph. Acta Cryst 2003;D59:519-21.

75. Charles JP. The regulation of expression of insect cuticle protein genes. Insect Biochem Mol Biol 2010;403:205-13.

76. Cheng T, Wu J, Wu Y, et al. Genomic adaptation to polyphagy and insecticides in a major East Asian noctuid pest. Nat Ecol Evol 2017;1:1747-56.

77. Pearce SL, Clarke DF, East PD, et al. Genomic innovations, transcriptional plasticity and gene loss underlying the evolution and divergence of two highly polyphagous and invasive Helicoverpa pest species. BMC Biol 2017;15:63.

78. Pashley DP. Host-associated differentiation in armyworms (Lepidoptera: Noctuidae): an allozymic and mitochondrial DNA perspective. In: Loxdale HD, der Hollander J, editors. Electrophoretic studies on agricultural pests. Oxford, UK: Oxford University Press; 1989. p. 103-14.

79. Rawlings ND, Barrett AJ. Families of serine peptidases. Method Enzymol 1994;244:19-61.

80. Feyereisen R. 2005. Insect cytochrome P450. In: Gilbert LI, Iatrou K, Gill SS, editors. Comprehensive molecular insect science. Vol 4, Biochemistry and molecular biology. Oxford: Elsevier. p. 1-77.

81. d'Alençon E, Sezutsu H, Legeai F, et al. Extensive synteny conservation of holocentric chromosomes in Lepidoptera despite high rates of local genome rearrangements. P NATL ACAD SCI USA 2010;107:7680-5.

82. Hill J, Rastas P, Hornett EA, et al. Unprecedented reorganization of holocentric chromosomes provides insights into the enigma of lepidopteran chromosome evolution. Sci Adv 2019;5:eaau3648. 
bioRxiv preprint doi: https://doi.org/10.1101/2019.12.26.889121; this version posted August 14, 2020. The copyright holder for this preprint

(which was not certified by peer review) is the author/funder, who has granted bioRxiv a license to display the preprint in perpetuity. It is made available under aCC-BY-NC 4.0 International license.

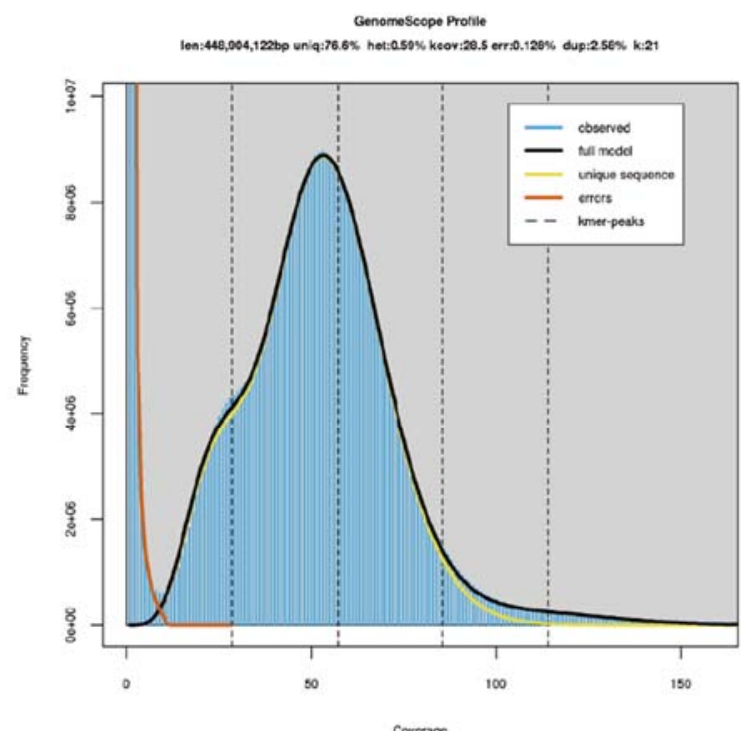

(a)

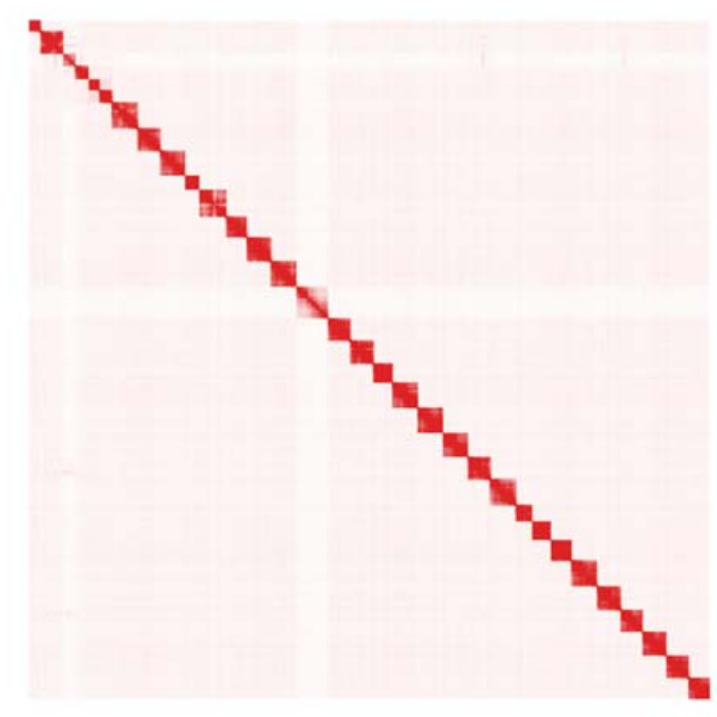

(b)

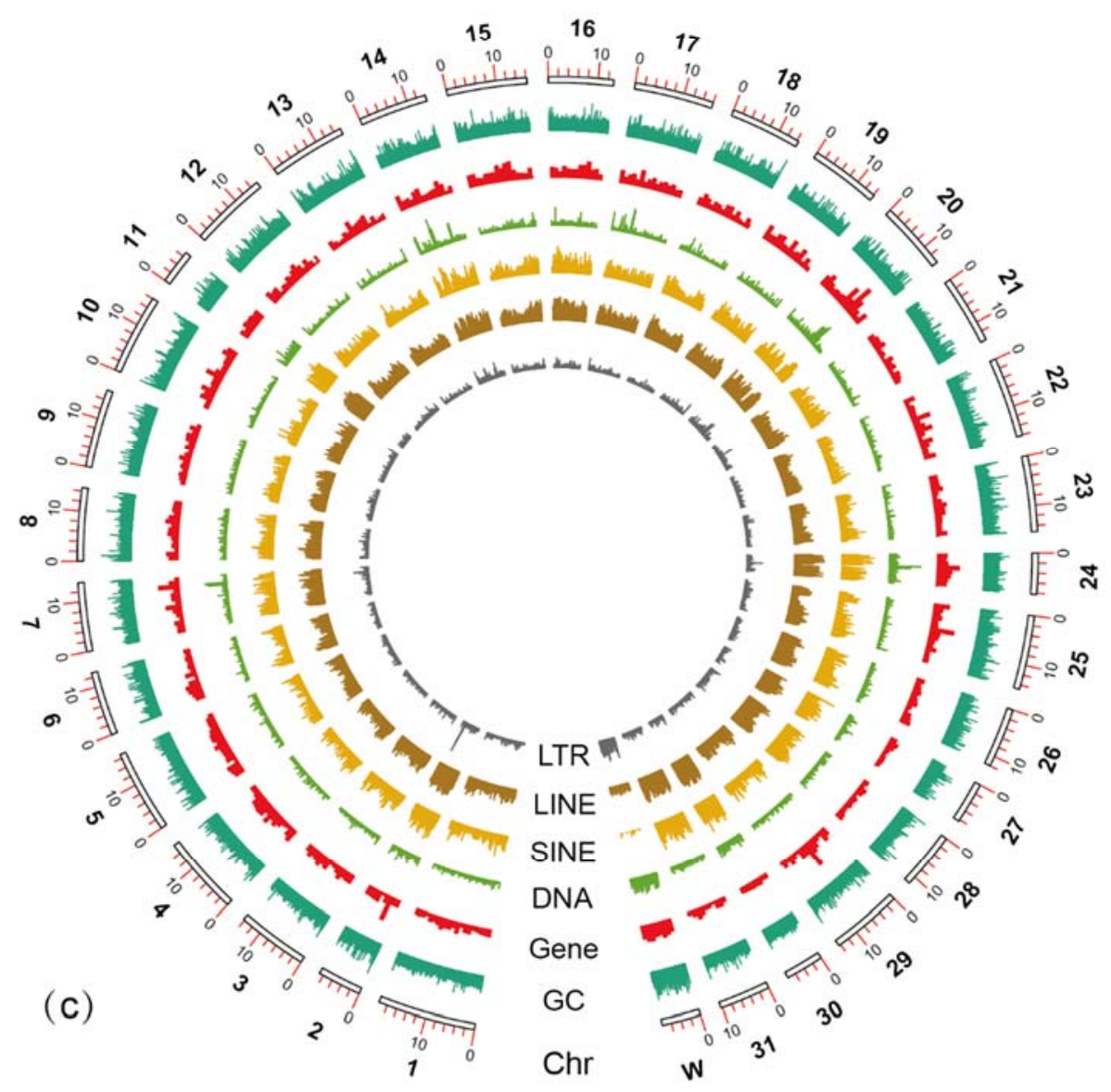


484 Figure 1: Genome characteristics of the beet armyworm genome. (a) Genome survey from 21-k-mer

485 frequency distribution plot estimated using GenomeScope (a maximum k-mer coverage of 10,000). (b) Hi-

486 C contact map with and among 32 curated pseudo-chromosomes (squares) generated from Juicebox. (c)

487 Circos tracks showing element distribution from outside to inside: GC content, gene density, DNA

488 transposons, SINEs, LINEs and LTRs; $10 \mathrm{~kb}$ sliding windows for GC content and $100 \mathrm{~kb}$ for others; the

489 most outer numbers indicating the chromosome nomenclature order and sizes $(\mathrm{Mb})$. 


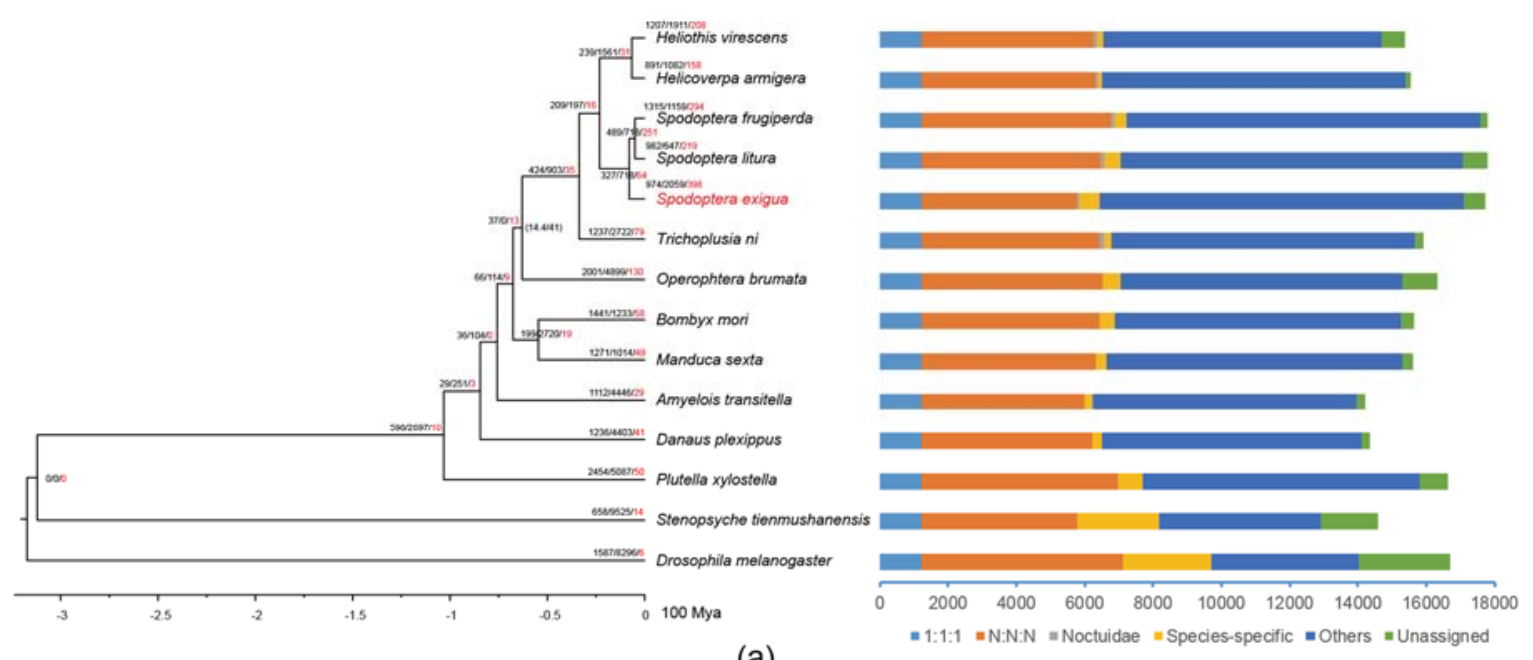

(a)

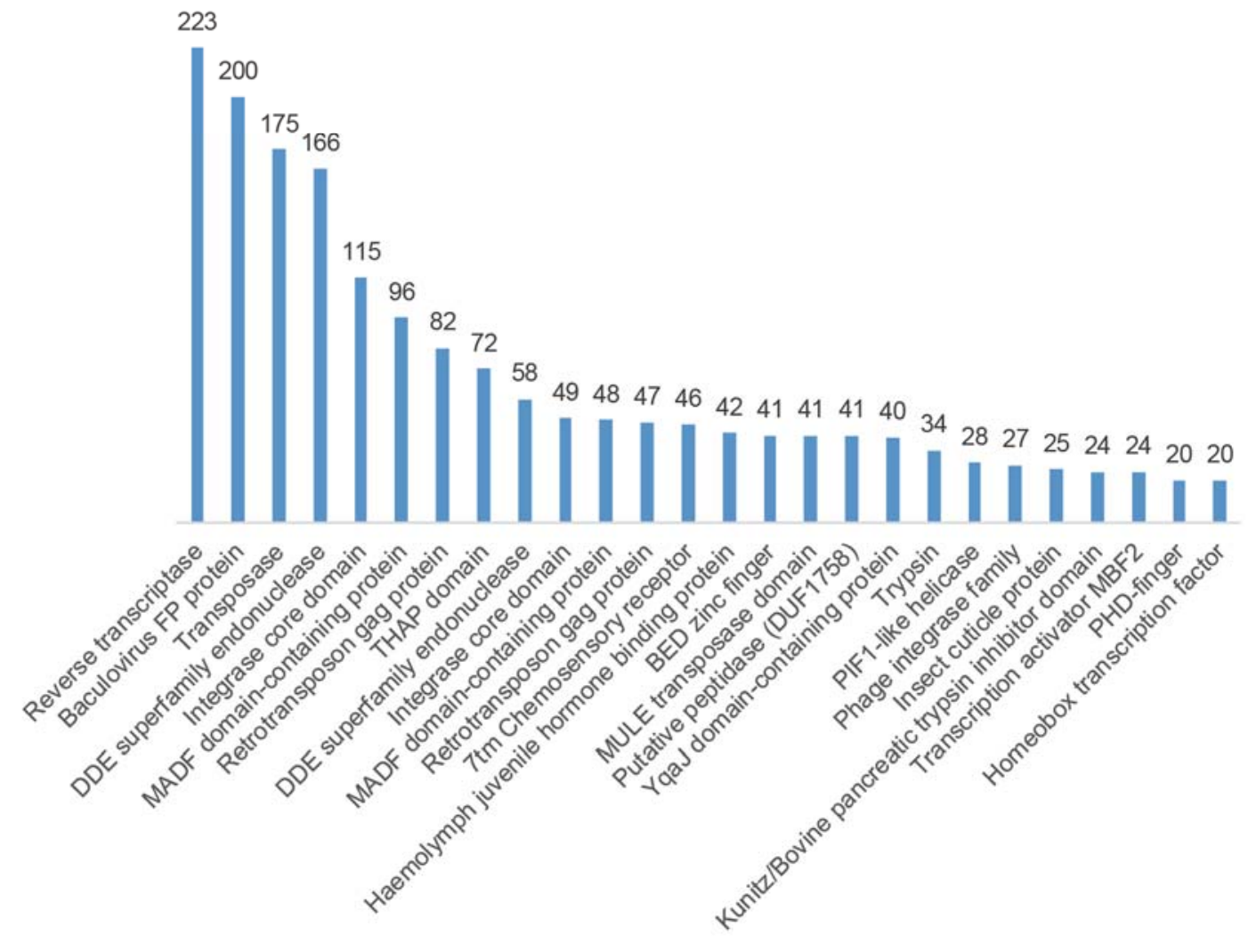

(b)

492 Figure 2: Phylogeny and gene family evolution. (a) Dating tree with node values representing the number of expanded, contracted and rapidly evolving families. Nodes support values fewer than 100 indicated within brackets. ' $1: 1: 1$ ' as shared single-copy genes, 'N:N:N' as orthologs from shared multi-copy orthogroups, 'Noctuidae' as shared orthologs unique to Noctuidae, 'Others' as unclassified orthologs, 'Unassigned' as orthologs which cannot be assigned into any orthogroups. (b) The significantly expanded 
bioRxiv preprint doi: https://doi.org/10.1101/2019.12.26.889121; this version posted August $14,2020$. The copyright holder for this preprint

(which was not certified by peer review) is the author/funder, who has granted bioRxiv a license to display the preprint in perpetuity. It is made available under aCC-BY-NC 4.0 International license.

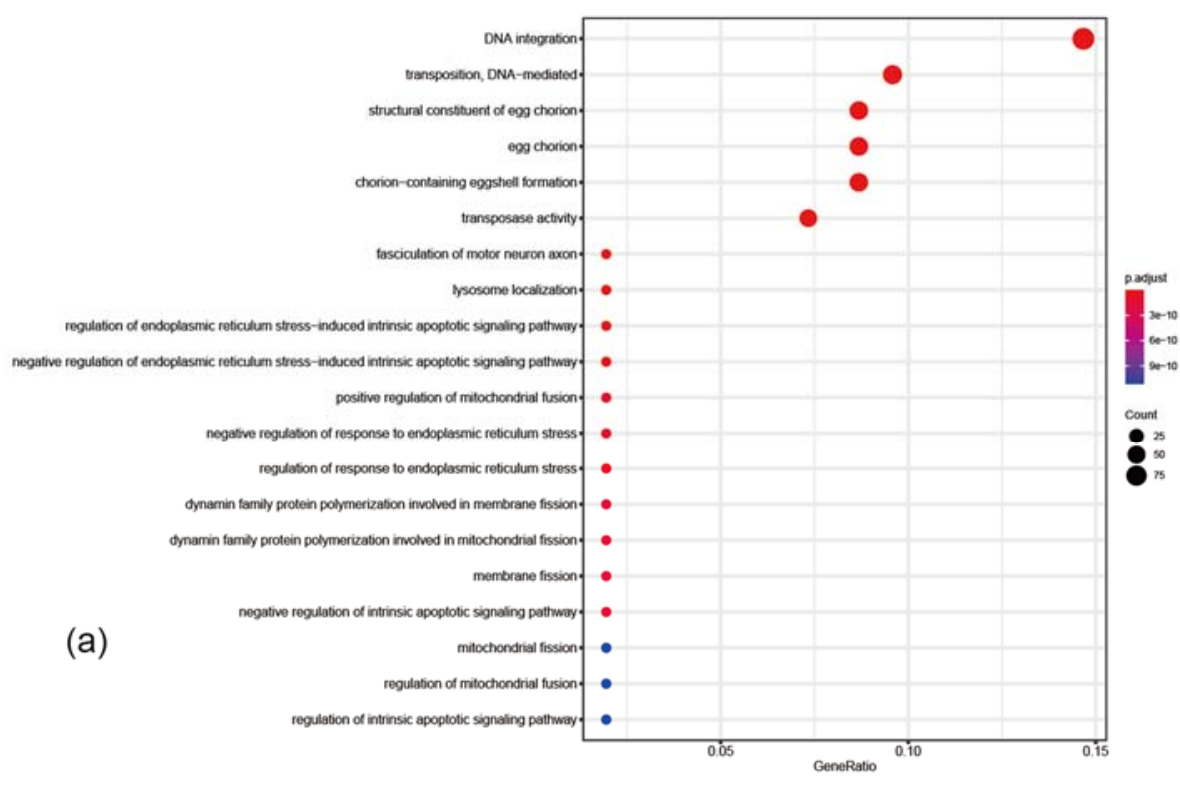

(b)

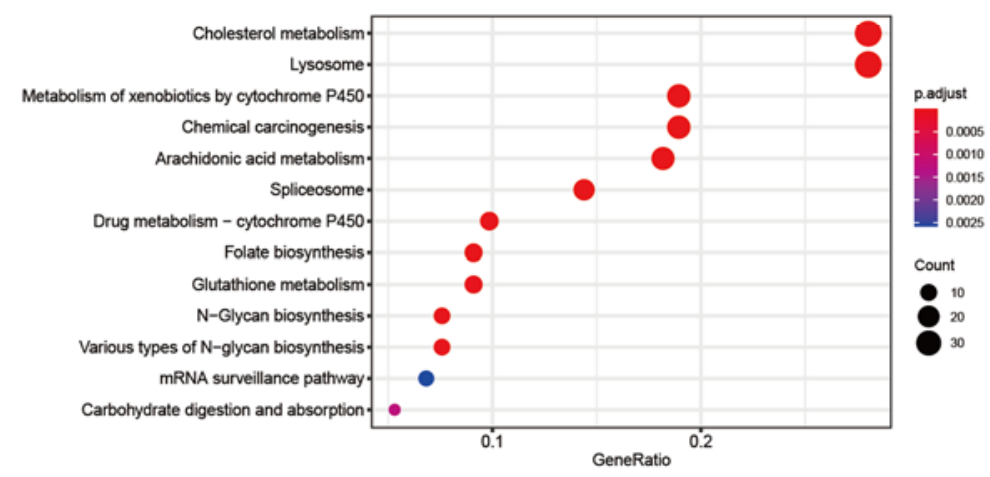

499

500 Figure 3: GO (a) and KEGG (b) function enrichment of significantly expanded gene families. Only the top

50120 categories shown. 

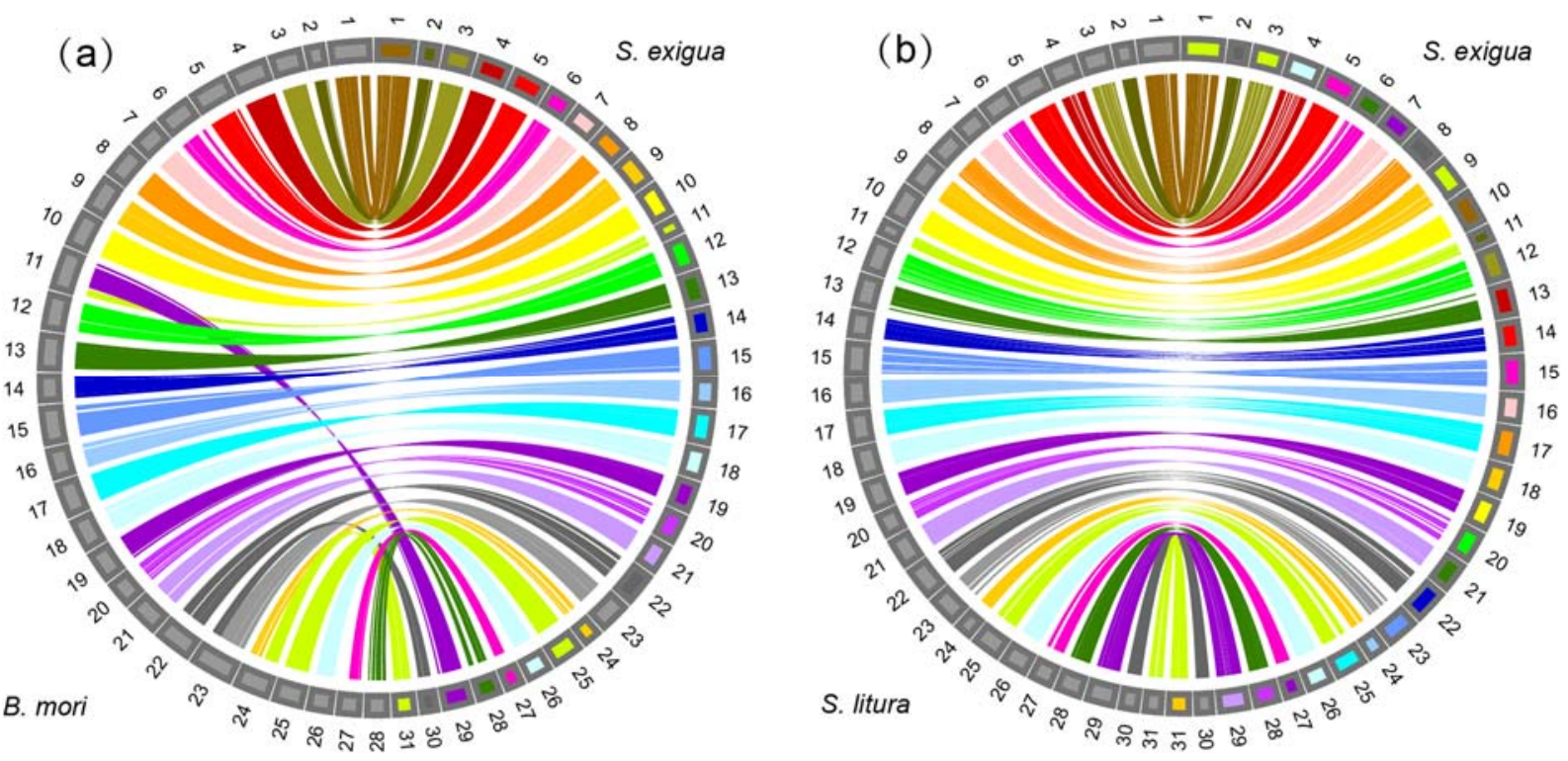

504 Figure 4: Circos plot of the synteny between the genomes. (a) S. exigua and B. mori. (b) S. exigua and S. 